\title{
Structural Characterization of Natural and Processed Zircons with X-Rays and Nuclear Techniques
}

\author{
Laura C. Damonte, ${ }^{1}$ Patricia C. Rivas, ${ }^{2}$ Alberto F. Pasquevich, ${ }^{3}$ Fernanda Andreola, ${ }^{4}$ \\ Federica Bondioli, ${ }^{5}$ Anna M. Ferrari, ${ }^{6}$ Laura Tositti, ${ }^{7}$ and Giorgia Cinellii \\ ${ }^{1}$ Departamento de Física, IFLP-CCT-CONICET, Facultad de Ciencias Exactas, Universidad Nacional de La Plata, \\ CC 67, 1900 La Plata, Argentina \\ ${ }^{2}$ Facultad de Ciencias Agrarias y Forestales, Universidad Nacional de La Plata, IFLP-CCT-CONICET, 60 y $119 \mathrm{~s} / \mathrm{n}$, \\ 1900 La Plata, Argentina \\ ${ }^{3}$ Departamento de Física, IFLP-CCT-CONICET, Facultad de Ciencias Exactas, Universidad Nacional de La Plata, \\ CICPBA, CC 67, 1900 La Plata, Argentina \\ ${ }^{4}$ Department of Engineering "Enzo Ferrari", University of Modena and Reggio Emilia, Via P. Vivarelli 10, 41125 Modena, Italy \\ ${ }^{5}$ Department of Engineering and Architecture, University of Parma, Parco Area delle Scienze 181/A, 43124 Parma, Italy \\ ${ }^{6}$ Department of Science and Methods of Engineering, University of Modena and Reggio Emilia, Via Amendola 2, \\ 42100 Reggio Emilia, Italy \\ ${ }^{7}$ Department of Chemistry "G. Ciamician”, University of Bologna, Via Selmi 2, 40126 Bologna, Italy
}

Correspondence should be addressed to Laura C. Damonte; lcdamonte@gmail.com

Received 20 March 2017; Accepted 18 June 2017; Published 10 September 2017

Academic Editor: Sergei Sergeenkov

Copyright (C) 2017 Laura C. Damonte et al. This is an open access article distributed under the Creative Commons Attribution License, which permits unrestricted use, distribution, and reproduction in any medium, provided the original work is properly cited.

In ceramic industry, zircon sand is widely used in different applications because zirconia plays a role as common opacifying constituent. In particular, it is used as a basic component of glazes applied to ceramic tiles and sanitary ware as well as an opacifier in unglazed bulk porcelain stoneware. Natural zircon sands are the major source of zirconium minerals for industrial applications. In this paper, long, medium, and short range studies were conducted on zirconium minerals originated from Australia, South Africa, and United States of America using conventional and less conventional techniques (i.e., X-Ray Diffraction (XRD), Positron Annihilation Lifetime Spectroscopy (PALS), and Perturbed Angular Correlations (PAC)) in order to reveal the type and the extension of the regions that constitute the metamict state of zircon sands and the modifications therein produced as a consequence of the industrial milling process and the thermal treatment in the production line. Additionally, HPGe gamma-ray spectroscopy confirms the occurrence of significant levels of natural radioactivity responsible for metamictization in the investigated zircon samples. Results from XRD, PALS, and PAC analysis confirm that the metamict state of zircon is a dispersion of submicron disordered domains in a crystalline matrix of zircon.

\section{Introduction}

Zirconium is the 18th most abundant element on Earth. In nature it mainly occurs as the free oxide $\mathrm{ZrO}_{2}$ (baddeleyite), but more commonly as zircon $\left(\mathrm{ZrSiO}_{4}\right)$. Mineral sands, the common name for zircon in connection with its most typical ore, are mainly exploited in the refractory and ceramic industries. The former application originates from zircon excellent thermophysical properties such as the low coefficient of thermal expansion, low electrical and thermal conductivity, and good corrosion resistance [1].

On the other hand, the widespread use of zircon sand in the ceramic industry ( $49 \%$ of the total consumption) depends on its use as a glazing and opacifying constituent. Natural zircon sands whose average grain size, for example, in granite rocks, is about $100-200 \mu \mathrm{m}$ are the major source of zirconium minerals for industrial applications; in this grain size range zircon can be used either as a feedstock to fused zirconia or in 
zirconium chemical plants as foundry sand or directly in the manufacture of some refractory materials. Other industrial applications require lower particle size usually restricted to two basic types: zircon flour $(40-50 \mu \mathrm{m})$ and micronized zircon $(D<5 \mu \mathrm{m})$ used as an opacifier [2,3]. The use of micronized zircon and zircon flour in ceramic products has been forecast to reach the $56 \%$ in 2012 of the total worldwide industrial consumption. Australia (the main producer), together with South Africa, United States, and India, accounts for over $80 \%$ of the global zircon production [3].

Beside about 2\% Hafnium (Hf), zircon sands typically hosts REEs (Rare Earth Elements) and relevant amount of naturally occurring radionuclides, namely, tetravalent uranium (U) and thorium (Th), replacing zirconium in the crystal lattice. Uranium and thorium concentration in zircon sands range, respectively, between 5-4000 ppm and 2-2000 ppm, depending on age and location of the deposits [4]. The levels of $U$ and Th in zircon sands are therefore frequently high enough to include these materials within the so-called NORMs, Naturally Occurring Radioactive Materials requiring handling and management according to appropriate radioprotection standards [3]. As parent nuclides of ${ }^{238} \mathrm{U},{ }^{235} \mathrm{U}$, and ${ }^{232} \mathrm{Th}$ radioactive families they are accompanied by their respective radioactive descendants which altogether contribute to significant radioactivity levels in zircons compared to average rocks. Cumulative radioactivity of zircon sands ranges between 5000 and $12000 \mathrm{~Bq} / \mathrm{kg}$ depending on ore age, weathering, and deposit location $[5,6]$. Due to radioactive decays of $U$ and Th families, in particular in the form of alpha radiation with energy $\geq 4,6 \mathrm{MeV}$ whose emission is accompanied by relevant recoil interactions, the structure of zircon can be heavily damaged over geological times, resulting in a partially aperiodic state, the so-called metamict state [7-9]. The metamict state can be described as a mixture of crystalline and amorphous domains whose extent depends on the dose of radiation absorbed by zircon $[8,9]$. For this reason exposure time, or, in other words, the age of zircons together with the enrichment in uranium and thorium, plays a relevant role in the degree of metamictization of each deposit. The amorphous domains due to radiation can be restored by recrystallization during thermal processes on geological times [10]. The crystalline and amorphous domains can be efficiently characterized by several instrumental techniques such as the widely employed electron microscopy (SEM [11], TEM [12]), X-Ray Diffraction (XRD) [8, 13], Raman spectroscopy [7], and, less frequently, Neutron Diffraction (ND) [14], while the structure of the domains damaged by irradiation is not well known due to the aperiodicity of the system $[10,15]$. The aperiodic or amorphous domains remain poorly studied since short range techniques of nanoscopic resolution are needed. The EXAFS experiments by Farges and Calas in metamict zircons from Madagascar and Japan show the coexistence of $\mathrm{Zr}$ (VII) and $\mathrm{Zr}$ (VIII) coordination, $\mathrm{Zr}$ (VIII) corresponding to the fundamental structure of the crystalline zircon and $\mathrm{Zr}$ (VII) produced by metamictization $[15,16]$. This observation allowed Farges to suggest that the metamict zircon structure could be supported by the existence of three different types of oxygen: (a) oxygen included in Zr-rich domains; (b) oxygen included in Si-rich domains; (c) oxygen as bridges among domains, $\mathrm{O}$ bridges being bound to two $\mathrm{Zr}$, and one $\mathrm{Si}$ as in the crystalline structure of zircon. Furthermore, Trachenko et al. [17] have studied the effects of radiation damage on structural changes in zircon by computer simulations, proposing damaged regions with nonuniform density, increasing it from depleted core to a densified boundary.

Further information on the surrounding of $\mathrm{Zr}$ and Hf both in natural zircons from Canada and in synthetic zircons and hafnon was collected by the determination of the quadrupole hyperfine interactions $[18,19]$. The remarkable sensitivity of the techniques employed in these studies allowed obtaining valuable in depth information, but the variability of situations and states presented by natural zircons due to their highly differentiated geological histories requires the contribution of extensive and demanding experimental research to fulfill statistical representativeness of zircon complexity.

In this work structural analysis of several zircon samples has been carried out using short range nuclear techniques, that is, Perturbed Angular Correlation (PAC) spectroscopy and Positron Annihilation Lifetime Spectroscopy (PALS) whose results were compared and integrated with those from the long-range X-Ray Diffraction. In particular XRD and PAC were used to both determine the amorphous fraction derived from cumulative alpha-decay events and analyze the atomic nanoconfigurations and defects of zircon sands [19]. The study covers the comparative analysis of sands of different origins (United States, Australia, and South Africa) with the aim of characterizing the type and the extension of the regions constituting the metamict state in untreated materials from geographically distinct areas as well as the modifications observed as a consequence of the industrial milling process and the thermal treatment (annealing) in the production line.

\section{Experimental Procedure}

The samples studied in the present work are natural zircons from the United States, Australia, and South Africa (provided by Endeka Ceramics) in two different stages of their industrial elaboration: sands (raw material) were named according to their origin as USA, AUS, and SUD, respectively (see Table 1 for chemical composition and granulometry), and sands micronized at a size of $D_{50}=4 \mu \mathrm{m}$ were named as USA4, AUS4, and SUD4. The milling process was conducted with an air-jet milling system (INCO) that is able to produce, without the help of moving and classifying parts, micronized particles typically $100 \%$ below $5 \mu \mathrm{m}$ (laser particle size analyzer, Mastersizer 2000, Malvern) without causing damage to the products. The micronized sands were subsequently annealed at $1000^{\circ} \mathrm{C}$ for $2 \mathrm{~h}$ in air atmosphere using an electric oven and the processed samples obtained will be indicated as aUSA4, aAUS4, and aSUD4, respectively.

2.1. Radioactivity Measurements. The radioactive content of the raw sand samples investigated and of the corresponding micronized derivatives was determined by high resolution $\gamma$-ray spectrometry using a HPGe p-type detector (Ortec, now Ametek). Aliquots of about $50 \mathrm{~g}$ of each sample were 
TABLE 1: Chemical composition (provided by Endeka Ceramics) for natural zircon sands. U and Th content and granulometry determined in natural and micronized zircon sands.

\begin{tabular}{|c|c|c|c|c|c|c|}
\hline & USA & USA4 & AUS & AUS4 & SUD & SUD4 \\
\hline $\mathrm{ZrO}_{2}+\mathrm{HfO}_{2}(\%)$ & 66.3 & & 62.2 & & 66.5 & \\
\hline $\mathrm{SiO}_{2}$ & 32.7 & & 33.3 & & 32.7 & \\
\hline $\mathrm{TiO}_{2}$ & 0.09 & & 0.15 & & 0.11 & \\
\hline $\mathrm{Al}_{2} \mathrm{O}_{3}$ & 0.58 & & 0.33 & & 0.18 & \\
\hline $\mathrm{Fe}_{2} \mathrm{O}_{3}$ & 0.058 & & 0.04 & & 0.06 & \\
\hline $\mathrm{Cr}_{2} \mathrm{O}_{3}$ & - & & - & & $<0.01$ & \\
\hline $\mathrm{CaO}$ & - & & - & & 0.01 & \\
\hline $\mathrm{U}(\mathrm{ppm})$ & $170_{10}$ & $144_{9}$ & $360_{22}$ & $311_{19}$ & $310_{19}$ & $336_{20}$ \\
\hline Th (ppm) & $67_{4}$ & $64_{4}$ & $219_{13}$ & $175_{11}$ & $143_{9}$ & $156_{9}$ \\
\hline Total activity $(\mathrm{Bq} / \mathrm{kg})$ & $13010_{1041}$ & $11390_{911}$ & $27840_{2227}$ & $23950_{1916}$ & $23600_{1880}$ & $26520_{2122}$ \\
\hline$D_{50}(\mu \mathrm{m})$ & 125 & 4.1 & 108 & 4.6 & 84 & 4.0 \\
\hline
\end{tabular}

accurately weighted and made up to a standard geometry in a polystyrene jar. All the samples were counted with the same procedure. The gamma spectra were acquired for 24 hours each in order to optimize radioactivity measurements. Qualitative and quantitative analysis of spectra were carried out relatively to a mixed radionuclide standard solution using the Ametek-Gamma Vision software; analytical results were checked by certified reference materials, namely, UTS-3 and DH1-a both by CANMET. Analysis of NORM $\gamma$-emitters was performed using a library of $\gamma$-emissions chosen to this scope based upon the highest photonic yields \% among those available for the members of all the three natural radioactive families [20]. ${ }^{238} \mathrm{U}$ concentration was determined by the ${ }^{226} \mathrm{Ra}$ emission at $186 \mathrm{keV}$ corrected for the ${ }^{235} \mathrm{U}$ interference, while the typical emissions of the ${ }^{214} \mathrm{Bi}$ and ${ }^{214} \mathrm{~Pb}$ daughters were taken independently, since they are likely influenced by loss of gaseous radon intermediate, which would lead to underestimation of the ${ }^{226} \mathrm{Ra}$ and ${ }^{238} \mathrm{U}$ [20]. ${ }^{232} \mathrm{Th}$ was determined through the ${ }^{228} \mathrm{Ac}$ emission at $338 \mathrm{keV}$. Concentrations in ppm for elemental uranium and thorium were determined by the following equations in the assumption of natural isotopic composition for the former element [21]:

$$
\begin{aligned}
& 1 \mathrm{ppm} \mathrm{U}=12.35 \mathrm{~Bq} / \mathrm{kg}^{238} \mathrm{U} . \\
& 1 \mathrm{ppm} \mathrm{Th}=4.072 \mathrm{~Bq} / \mathrm{kg}^{232} \mathrm{Th} .
\end{aligned}
$$

2.2. XRD Analysis. The X-Ray Diffraction spectra were recorded at room temperature using $\mathrm{Cu}-\mathrm{K}_{\alpha}$ radiation in a Philips PW1700 diffractometer. Quantitative analysis of the samples was performed by the combined Rietveld RIR (Reference Intensity Ratio) method. $10 \mathrm{wt} \%$ of corundum (NIST SRM $674 \mathrm{a}$ annealed at $1500^{\circ} \mathrm{C}$ for 1 day to increase the crystallinity to $100 \mathrm{wt} \%$ ) was added to all samples as an internal standard. The mixtures, ground in an agate mortar, were side-loaded in an aluminum flat holder in order to minimize the preferred orientation problems. Data were recorded in the $5^{\circ}-140^{\circ} 2 \theta$ range (step size $0.02^{\circ}$ and $6 \mathrm{~s}$ counting time for each step). The phase fractions extracted by the Rietveld RIR refinements, using GSAS software and EXPGUI as graphical interface, were rescaled on the basis of the absolute weight of corundum originally added to the mixtures as an internal standard and therefore internally normalized [22]. The background was successfully fitted by a Chebyshev function with a variable number of coefficients depending on its complexity. The peak profiles were modeled using a pseudo-Voigt function with one Gaussian (GW) and two Lorentzian $(L x, L y)$ coefficients. Lattice constants, phase fraction, and coefficients corresponding to sample displacement and asymmetry were also refined. The cut-off value for the calculation of the peak profiles in all refinements was $0.05 \%$.

2.3. PALS Analysis. Positron Annihilation Lifetime Spectroscopy (PALS) is based on the capability of positron to sense the electron density in the material; in consequence it is highly sensitive to open volumes in solids. In matter, a positron and an electron mainly annihilate by the emission of two gamma rays of $511 \mathrm{keV}$. Under given circumstances, both particle and antiparticle can form a bound state, called positronium (Ps) [23]. The ortho-positronium (o-Ps, the spins of the two particles are parallel oriented) is commonly annihilated with an electron of the medium to give two gamma radiations. The measured positron and o-Ps lifetimes are related to the open volume defect size allowing characterizing the defect structure of the material under study. PALS measurements were done in a conventional fast-fast coincidence system with two scintillator detectors (one $\mathrm{BaF}_{2}$ and one plastic BURLE) provided a time resolution (FWHM) of 260 ps. $\mathrm{A}^{22} \mathrm{NaCl}(10 \mu \mathrm{Ci})$ radioactive source deposited onto a kapton foil $\left(1.42 \mathrm{~g} / \mathrm{cm}^{3}\right)$ and sandwiched between two identical samples was used. The analyzed sands were uniaxial pressed into $8 \mathrm{~mm}$ diameter pellets of around $1 \mathrm{~mm}$ thick. The source contribution and the response function were evaluated from an Hf metal reference sample using the RESOLUTION code [24]. Positron lifetime spectra of $3 \times 10^{6}$ counts each were recorded at room temperature and analyzed with the POSITRONFIT program [24]. After background and source contribution correction, the lifetime spectra

$$
n(t)=\sum_{i} I_{i} e^{-t / \tau_{i}}
$$


were decomposed into different exponential decays, each positron state being characterized by a positron lifetime, $\tau_{i}$, with certain intensity, $I_{i}$ (normalized).

2.4. PAC Analysis. The Perturbed Angular Correlation (PAC) method is based on the hyperfine interactions of nuclear moments with extra nuclear fields. This technique provides a nanoscopic (i.e., short range) description of the Zr-ions nearest neighborhoods. The nuclei of ${ }^{181} \mathrm{Hf}$, obtained by irradiation with thermal neutrons (RA3-reactor of the Comision Nacional de Energía Atómica (CNEA)), of the ${ }^{180} \mathrm{Hf}$ natural impurities located at $\mathrm{Zr}$ sites constitute the radioactive probe nuclei in the zircon powders. The $\gamma-\gamma$ cascade at (133-482) $\mathrm{keV}$, populated by the $\beta$-decay of ${ }^{181} \mathrm{Hf}$, was used to measure the quadrupole interaction of the $482 \mathrm{keV}(+5 / 2)$ state of ${ }^{181} \mathrm{Ta}$. The time differential anisotropy was calculated from the coincidence spectra $N(\theta, t)$, where $\theta$ is the angle between detectors and $t$ is the time delay between the two gamma events. After subtraction of chance coincidence background, time spectra corresponding to angles of $90^{\circ}$ and $180^{\circ}$ between detectors were combined to obtain the ratio

$$
R(t)=2 \frac{N\left(180^{\circ}, t\right)-N\left(90^{\circ}, t\right)}{N\left(180^{\circ}, t\right)+2 N\left(90^{\circ}, t\right)}=A_{22} G_{22}(t) .
$$

The $G_{22}(t)$, the perturbation function, for a static quadrupole interaction has the following expression:

$$
G_{22}(t)=\sum_{n=0}^{3} S_{2 n} e^{-\delta \omega_{n} t} \cos \left(\omega_{n} t\right) .
$$

The frequencies, $\omega_{n}$, are related to the quadrupole frequency $\omega_{\mathrm{Q}}=\pi e Q V_{Z Z} / 20 h$, by $\omega_{n}=g_{n}(\eta) \omega_{\mathrm{Q}}$. The $g_{n}(\eta)$ coefficients are known functions of the asymmetry parameter, $\eta$, defined by $\eta=\left(V_{x x}-V_{y y}\right) / V_{z z}$, where $V_{i i}$ are the principal components of the EFG tensor. The exponential function takes into account a Lorentzian frequency distribution of width $\delta$ around the $\omega_{\mathrm{Q}}$ values due to disorder and/or impurities or defects present in the lattice.

On account of the $r^{-3}$ dependence of the quadrupole hyperfine interaction, the technique is extremely localized and different surroundings can be determined. Thus, a relative fraction or intensity $\left(f_{i}\right)$ and the corresponding EFG parameters $\left(\omega_{\mathrm{Q} i}, \eta_{i}, \delta_{i}\right)$ can be determined for each neighborhood. Finally, the experimental ratio $R(t)$ is fitted by $R(t)=A_{22} \sum f_{i} G_{22}^{i}(t)$, where $f_{i}$ are the relative fraction of nuclei experiencing a given perturbation $G_{22}^{i}(t)[25,26]$.

PAC measurements were performed at room temperature using a standard setup with four conical $\mathrm{BaF}_{2}$ scintillation detectors with a time resolution of $0.6 \mathrm{~ns}$ (FWHM). Details on the experimental setup adopted in this work can be found elsewhere [27].

\section{Results and Discussion}

The $\mathrm{U}$ and $\mathrm{Th}$ contents determined in the investigated samples (see Table 1) are comparable to those published by Bruzzi et al. [5] and to those regularly checked by the authors from Bologna University in the long term collaboration
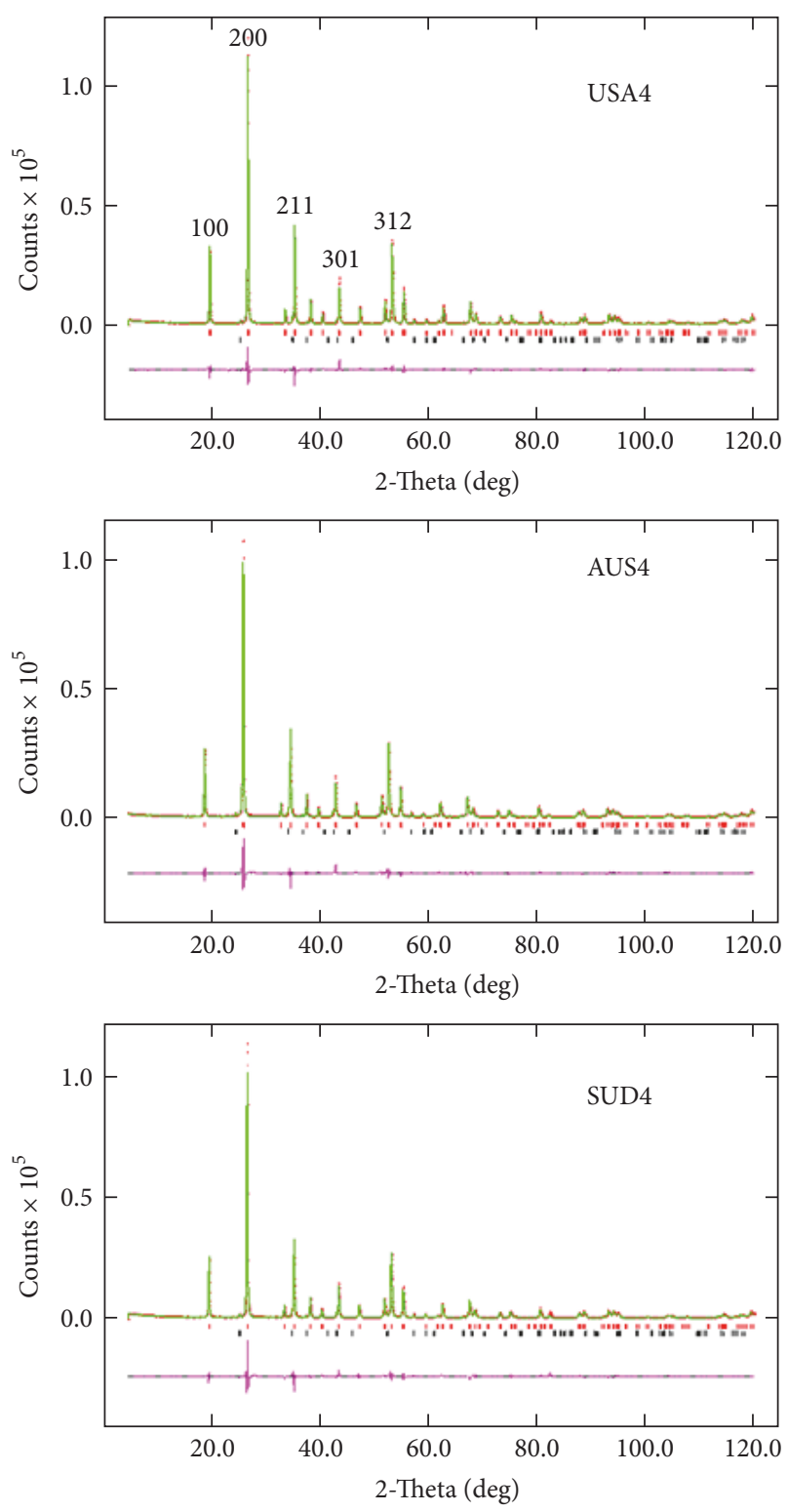

FIGURE 1: Rietveld refinements results for micronized zircon sands. Points are the experimental data while full line is the results from the fits. Column bars below represent peak position for zircon and corundum phases. The full line below represents the difference from data to refinement.

with important ceramic companies of the Modena Ceramic district. Consequently the NORM levels detected suggest that the zircons analyzed in this work are partially metamicted.

The XRD patterns obtained in natural zircon sands only reveal the principal lines corresponding to $\mathrm{ZrSiO} 4$. Rietveld RIR refinements were performed on natural and micronized samples. The obtained cell parameters and quantitative analysis are shown in Table 2 while Figure 1 shows the refinement results for the micronized samples. A small decrease in lattice parameters is observed with respect to the standard $\mathrm{ZrSiO} 4$, probably consistent with the presence of interstitial defects typical of metamictic state. In agreement with $U$ and $T h$ 
TABLE 2: Results of Rietveld quantitative analysis (wt\%) RIR. Standard parameters for $\mathrm{ZrSiO} 4$ are from literature (ICSD 9582).

\begin{tabular}{lccccrr}
\hline Sample & $\mathrm{ZrSiO}_{4}$ & Amorphous & $a=b$ & $c$ & $\chi^{2}$ & $R_{\mathrm{wp}}$ \\
\hline USA & $49.9_{1}$ & $50.1_{2}$ & $6.6059_{1}$ & $5.9878_{1}$ & 1.884 & 0.089 \\
USA4 & $66.8_{2}$ & $33.2_{4}$ & $6.6058_{1}$ & $5.9864_{1}$ & 3.347 & 0.069 \\
AUS & $48.5_{1}$ & $51.5_{2}$ & $6.6075_{1}$ & $5.9927_{1}$ & 2.182 & 0.095 \\
AUS4 & $59.1_{1}$ & $40.8_{3}$ & $6.6077_{1}$ & $5.9924_{1}$ & 3.675 & 0.124 \\
SOU & $39.7_{1}$ & $60.3_{1}$ & $6.6066_{1}$ & $5.9918_{2}$ & 2.054 & 0.093 \\
SOU4 & $57.6_{1}$ & $42.4_{4}$ & $6.6072_{1}$ & $5.9925_{1}$ & 3.587 & 0.091 \\
$\mathrm{ZrSiO}_{4}$ & & & $6.612_{2}$ & $5.994_{2}$ & & 0.123 \\
\end{tabular}

TABLE 3: Hyperfine quadrupole parameters fitted to the experimental PAC results. Errors are indicated as subindexes.

\begin{tabular}{|c|c|c|c|c|c|}
\hline Sample & $\begin{array}{c}\text { Relative fraction } \\
f(\%)\end{array}$ & $\begin{array}{l}\text { Quadrupole frequency } \\
\omega_{Q}(\mathrm{Mrad} / \mathrm{s})\end{array}$ & $\begin{array}{l}\text { Asymmetry } \\
\text { parameter } \eta\end{array}$ & $\begin{array}{c}\text { Frequency distribution } \\
\text { width } \delta(\%)\end{array}$ & $\begin{array}{c}\text { Interaction } \\
\text { label }\end{array}$ \\
\hline \multirow{4}{*}{ USA } & $49_{1}$ & $101_{1}$ & $0.06_{1}$ & $3_{1}$ & $I_{1}$ \\
\hline & $21_{1}$ & $109_{1}$ & $0.87_{1}$ & $7_{1}$ & $I_{2}$ \\
\hline & $22_{1}$ & $76_{1}$ & $0.62_{2}$ & $12_{1}$ & $I_{3}$ \\
\hline & $88_{1}$ & $191_{1}$ & $0.73_{2}$ & $3_{1}$ & $I_{4}$ \\
\hline \multirow{4}{*}{ AUS } & $40_{1}$ & $101_{1}$ & $0.09_{1}$ & $4_{1}$ & \\
\hline & $35_{1}$ & $104_{1}$ & $0.78_{2}$ & $15_{2}$ & \\
\hline & $16_{1}$ & $66_{1}$ & $0.77_{4}$ & $11_{4}$ & \\
\hline & $99_{1}$ & $190_{2}$ & $0.65_{2}$ & $4_{1}$ & \\
\hline \multirow{4}{*}{ SUD } & $47_{1}$ & $101_{1}$ & $0.10_{1}$ & $5_{1}$ & \\
\hline & $34_{1}$ & $105_{1}$ & $0.73_{1}$ & $11_{1}$ & \\
\hline & $11_{1}$ & $67_{1}$ & $0.77_{3}$ & $5_{1}$ & \\
\hline & $8_{1}$ & $191_{2}$ & $0.62_{2}$ & $4_{1}$ & \\
\hline
\end{tabular}

content (Table 1) USA sand is the most crystalline material as compared with the other two samples investigated. In addition, the milling process reduced grain size and amorphous content (Tables 1 and 2), without changing cell parameters.

The lifetime components resolved in the PALS spectra differ in the natural and processed zircon (Figure 2). The evolution of the spectra appears to be consistent with the structure of natural zircon where the longest lifetimes $(\tau=$ $1800 \mathrm{ps}$ ) originate from positronium annihilating in cavities. The lowest lifetimes present both in natural and in processed zircon seem rather to correspond to positron annihilating in zircon crystalline bulk ( $\tau=300 \mathrm{ps})$ or in vacancy-type defects of smaller size ( $\tau=500 \mathrm{ps})$ than the cavities where positronium annihilates.

If the formation of o-positronium in the cavities of the natural sands is assumed, using the semiempirical relation between the o-positronium half-life $\tau$ (ns) and the average cavity dimension $R(\AA)$ where it is annihilated [28]

$$
\tau=\frac{1}{2}\left[1-\frac{R}{R+1.66}+\frac{1}{2 \pi} \sin \left(\frac{2 \pi R}{R+1.66}\right)\right]^{-1}
$$

it is possible to estimate that the natural sands cavities are approximately of $80 \AA^{3}$ ( $\tau \cong 1800 \mathrm{ps}$ ).
As a consequence of the micronization process the cavities disappear and only crystalline materials with regions displaying intrinsic defects coexist. Further annealing heals the defects, leading to a crystalline state (Figure 2(c)).

The results of the quantitative Rietveld RIR analysis are in good agreement with PALS observations. Table 2 shows that the amorphous content decreases around 30\% from zircon natural sands to the micronized ones favoring the crystalline $\mathrm{ZrSiO}_{4}$ phase.

The PAC spectra (Figure 3(a)) and their associated Fourier transform (Figure 3(b)) for natural sands are typical for polycrystalline samples. All of them were analyzed using four quadrupole components according to the perturbation factor (3) (see Table 3).

Fitting the PAC spin rotation curves both for micronized sands and for those thermally treated revealed different combinations of the four interactions determined in the natural sands. The whole evolution of the relative fractions obtained for all the samples is shown in Figure 4. Relative to the micronization of the sands, PAC shows no changes in the amounts of the interactions. The thermal annealing of the micronized sands instead indicates a remarkable increase of the fraction $f$ for interaction $I_{1}$; in addition, a systematic decrease of $I_{2}$ fraction is observed. The decrease is higher for the SUD than for the USA and AUS zircon sands. 

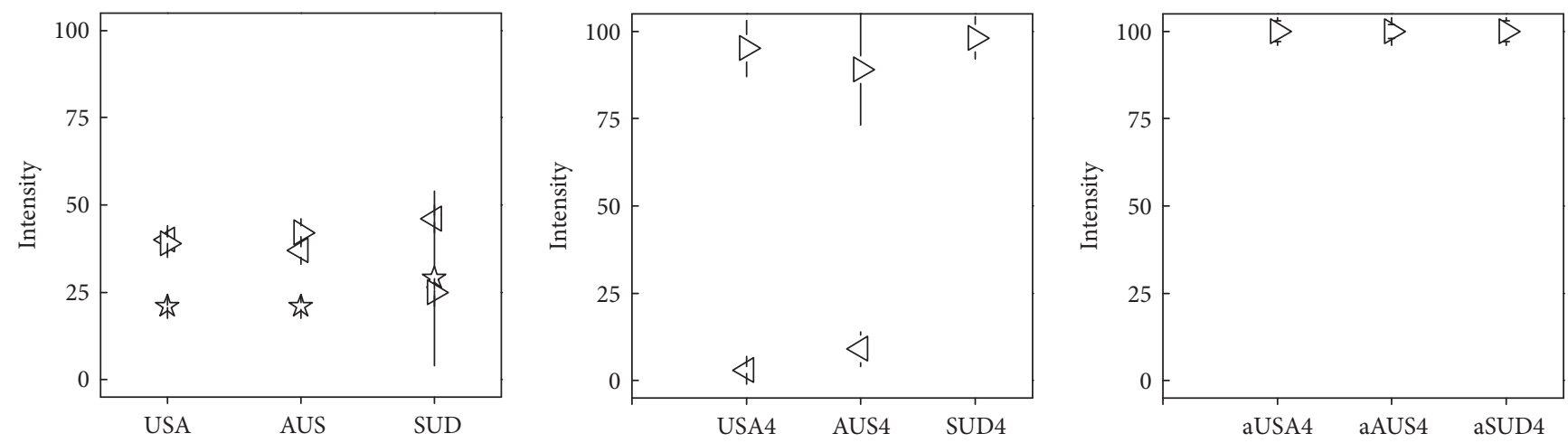

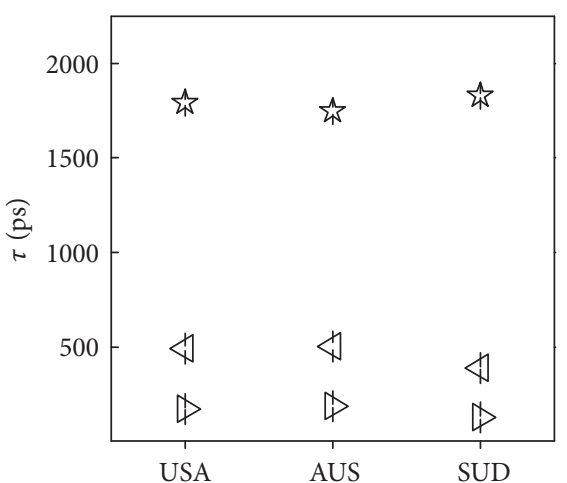

(a)

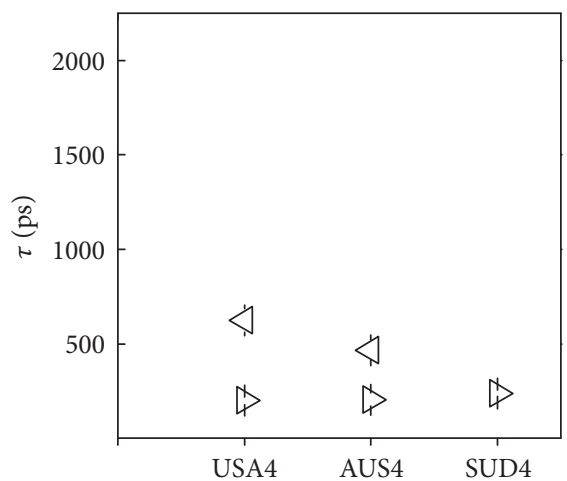

(b)

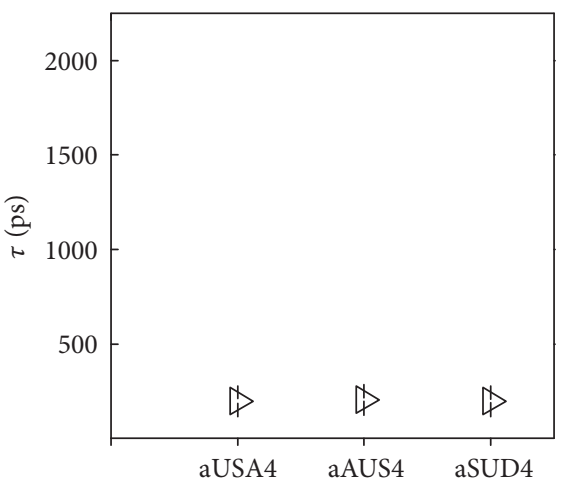

(c)

Figure 2: Lifetime components and their intensities determined by PALS for the natural (a), the micronized (b), and the annealed micronized sands (c).

The comparison of the $f$ values in Table 3 and Figure 4 shows that the most relevant hyperfine interaction $\left(I_{1}, \omega_{\mathrm{Q} 1}\right.$ $=101 \mathrm{Mrad} / \mathrm{s}$ and $\left.40<f_{1} \%<49\right)$ corresponds to $\mathrm{Zr}$ lattice site in crystalline zircons in agreement with quadrupole frequency values reported previously [19, 27]. With significant relative abundance $\left(20<f_{2} \%<35\right)$ very asymmetric and slightly distributed interaction $I_{2}$ of quadrupole frequency $\left(\omega_{\mathrm{Q} 2}=104-109 \mathrm{Mrad} / \mathrm{s}\right)$ similar to that of the crystalline zircon is also observed in the analyzed samples. This interaction has been observed in fully metamict zircons $[19,29]$. The above-mentioned interactions are accompanied by the interactions of similar or lower abundance $I_{3}\left(11<f_{3} \%<\right.$ $22)$ and $I_{4}\left(8<f_{4} \%<9\right)$. PAC studies on synthetic zircon and mullite-zirconia system [27] determined the presence of a similar $\mathrm{I}_{3}$ interaction associated with aperiodic regions. This interaction which exists in synthetic zircons cannot be attributed to the metamict state. Interaction $I_{4}$ has not been reported in natural zircons so far. Thus, PAC results show that the metamict state of zircon may consist in an atomic arrangement with two different $\mathrm{Zr}$ surroundings described by $I_{2}$ and $I_{4}$ interactions. Furthermore, PAC allows validating the model of the metamict state of three regions (a Si-rich region, a Zr-rich region, and a bridge region) suggested by Farges [15] since the model admits two nonequivalent sites for the $\mathrm{Zr}(\mathrm{VII})$. The fact that the $I_{2}$ interaction has a quadrupole frequency similar to that of crystalline zircon $\left(I_{1}\right)$ but more asymmetric and slightly distributed gives an experimental support to assigned it to $\mathrm{Zr}$ probes involved in the bridge zone since they resemble the zirconium environment in the crystalline zircon. On the other hand, quadrupole parameters of the $I_{4}$ interaction are close to those reported for the $\mathrm{Zr}$ (VII) in the tetragonal metastable form of $\mathrm{ZrO}_{2}$ [30]; therefore this interaction can be directly associated with the $\mathrm{Zr}$-rich regions proposed in the model. Simple calculations of the quadrupole interaction based on the zircon structure, using the point charge model [31] for the first $\mathrm{Zr}$ coordination, provide additional support since they suggest the existence of interactions of high asymmetry parameter $\eta$ for configurations of one oxygen vacancy in the $\mathrm{ZrO}_{8}$ group.

Summing up, in terms of the model proposed by Farges [15] for the metamict state of the zircon, it is possible to assign the following interpretation to the quadrupole interactions observed in the described experiment:

(i) $I_{1}$ and $I_{3}$ interactions are the corresponding ones to probes immersed in crystalline zircon $\left(I_{1}\right)$ that coexist with aperiodic zircon $\left(I_{3}\right)$.

(ii) $I_{2}$ and $I_{4}$ correspond to two nonequivalent zirconium sites of the metamict zircon in Zr-rich regions $\left(I_{4}\right)$ and in the bridge region that contains $\mathrm{Zr}$ and $\mathrm{Si}\left(I_{2}\right)$.

In this circumstance, the addition of the relative fractions of $I_{2}$ and $I_{4}$ represents the extent or degree of metamictization 

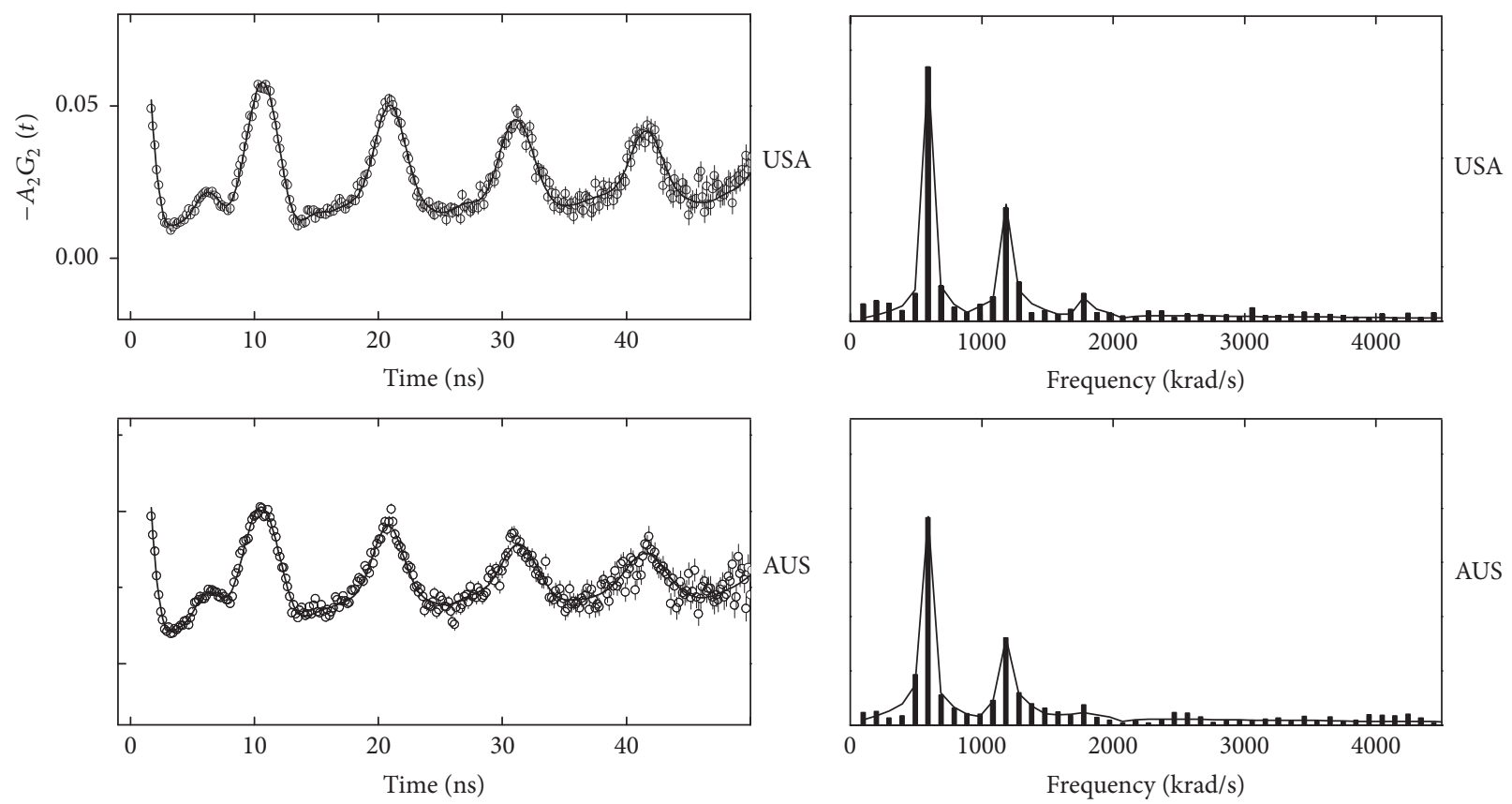

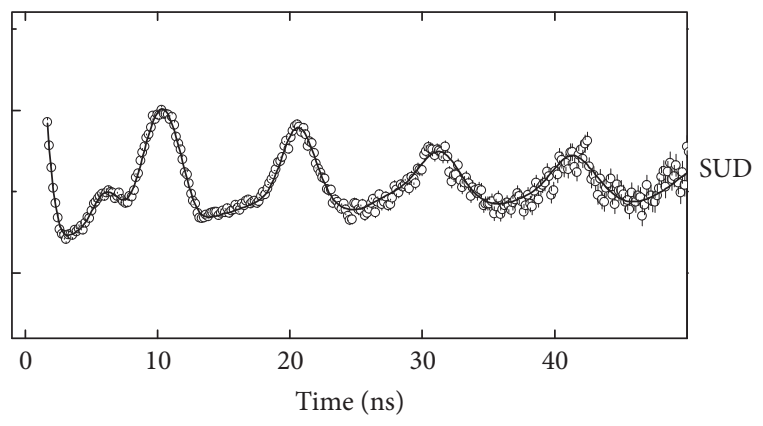

(a)

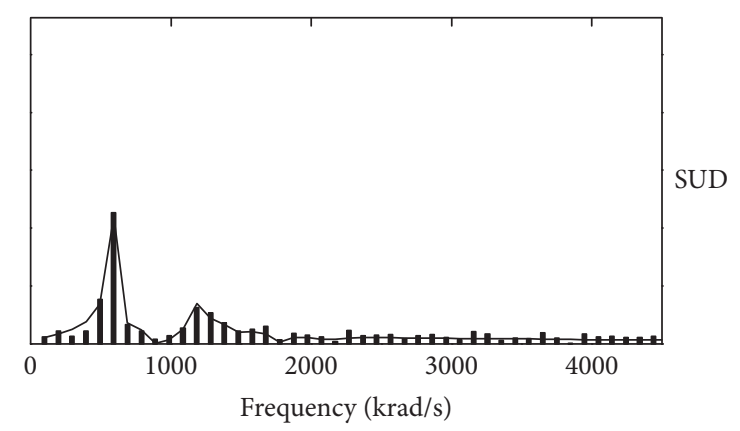

(b)

Figure 3: PAC spectra for zircon sands. The experimental spin rotation curves $A_{2} G_{2}(t)$ are shown on (a); the Fourier transform amplitude spectra are plotted on (b). Solid lines represent the least-squares-fitted PAC spectra and their respective Fourier transforms obtained with the values given in Table 3 for the fitting parameters.

of the zircons: 29,44 , and $42 \%$ for USA, AUS, and SUD, respectively (see Table 3 ). The values show a reasonable agreement with those expected from the content of radioactive elements determined in the sands (see Table 1) and consequently with radiation dose [9]. According to Farges and modeling the $\mathrm{Zr}$-rich region as the core of a sphere surrounded by the bridge region as shown in Figure 5, it is possible to estimate an order of a magnitude for the size of the metamict regions based on the relative abundance of the $I_{2}$ and $I_{4}$ interactions. In fact, the ratio of the relative fractions $f_{2} /\left(f_{2}+f_{4}\right)$ is proportional to the ratio between the volume containing PAC probes experiencing $I_{2}$ interaction and the volume containing all PAC probes of the metamict zone. This last volume ratio

$$
100\left[1-\left(\frac{R-r}{R}\right)^{3}\right]
$$

is plotted in Figure 6 versus the bridge region thickness $r$, for different spheres sizes $R$. If we assume that the metamictic region has a radius of about $40 \AA$, similar to other author's proposals (Weber et al. 1999 and [17]), our experimental results of the ratio $f_{2} /\left(f_{2}+f_{4}\right)$, shown in Table 4, allow estimating the width bridge zone $r$ in about $15 \AA$ (dashed sectors of Figure 6).

The three applied techniques, XRD, PALS, and PAC, report similar degree of metamictization for all natural sands in agreement with the determined $\mathrm{U}$ and $\mathrm{Th}$ content.

The proposed model based on PAC results to describe the metamictic region is consistent with the presence of the small cavities determined by PALS within the $\mathrm{Zr}$-rich region which is the less dense one. This feature is also in agreement with the computer simulations reported by Trachenko et al. [17].

A gradual diminution of the metamictic state as processing advances (micronized and annealed samples) is observed by all the techniques. However, while XRD and PALS show an apparent complete recovery of the crystalline state, PAC senses residual $\mathrm{Zr}$ environments inside the metamictic state. 
TABLE 4: Relative abundances representing the amount of PAC probes in the bridge region $\left(f_{2}\right)$ relative to all the PAC probes in the metamict region $\left(f_{2}+f_{4}\right)$.

\begin{tabular}{lccc}
\hline Geological origin of the samples & Natural sand & Micronized sand & Annealed micronized sand \\
\hline USA & 74 & 71 & 59 \\
AUS & 80 & 80 & 87 \\
SUD & 80 & 76 & 73 \\
\hline
\end{tabular}
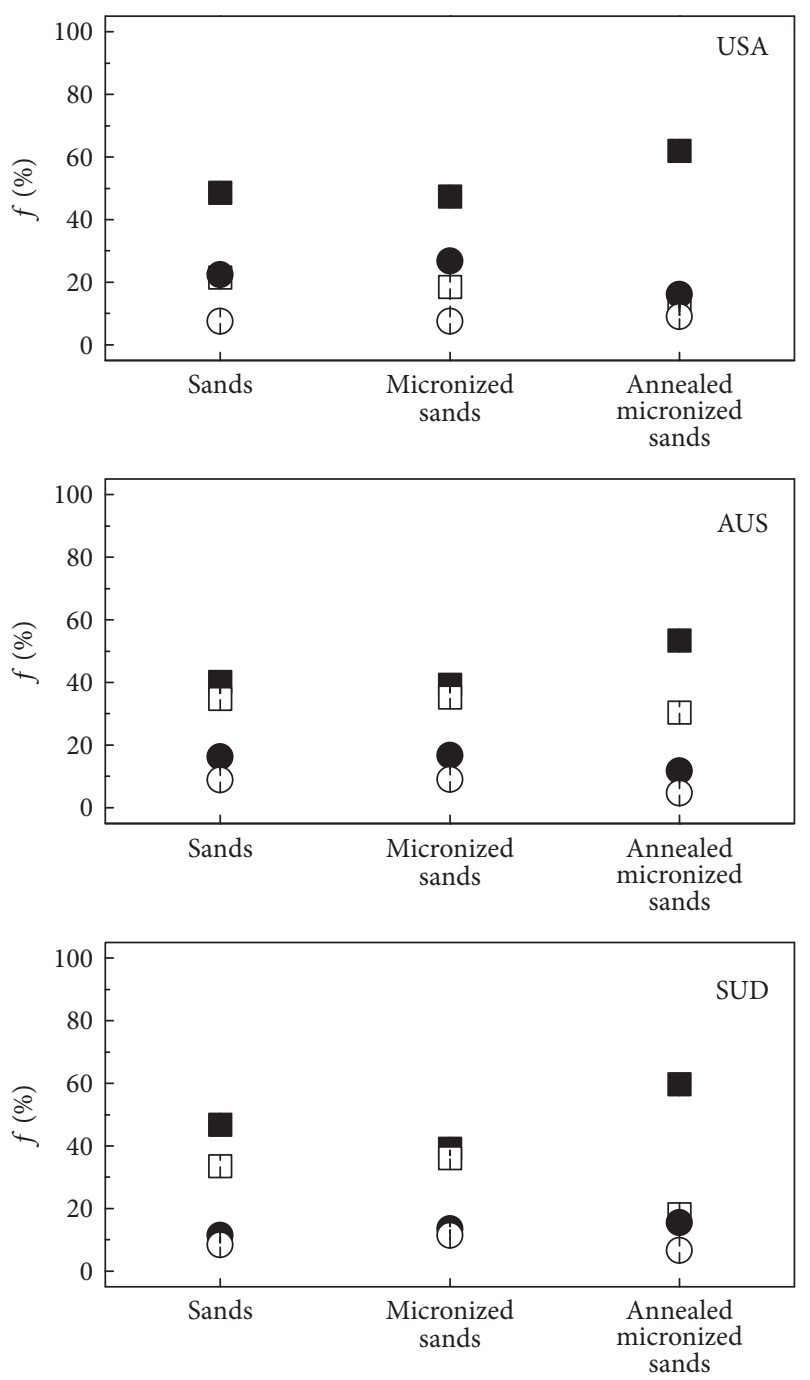

FIGURE 4: PAC relative fractions evolution determined for the natural, the micronized, and the annealed micronized zircon sands. Symbols represent the relative fractions of the four hyperfine interactions reported in Table 3: $I_{1}$ (full square), $I_{2}$ (open square), $I_{3}$ (full circle), and $I_{4}$ (open circle).

\section{Conclusions}

In this paper long, medium, and short range studies (X-Ray Diffraction (XRD), Positron Annihilation Lifetime Spectroscopy (PALS), and Perturbed Angular Correlations, (PAC)) were successfully applied to characterize the zirconium minerals originated from Australia, South Africa, and United States of America. It was established that the metamictization

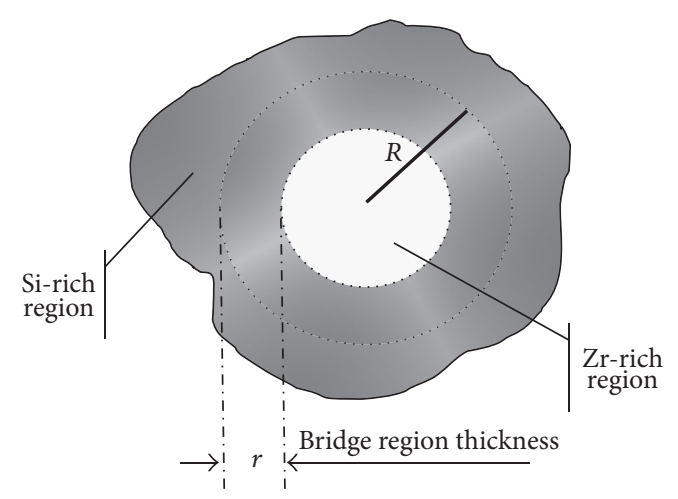

FIGURE 5: Schematic representation of metamict state as proposed by the authors.

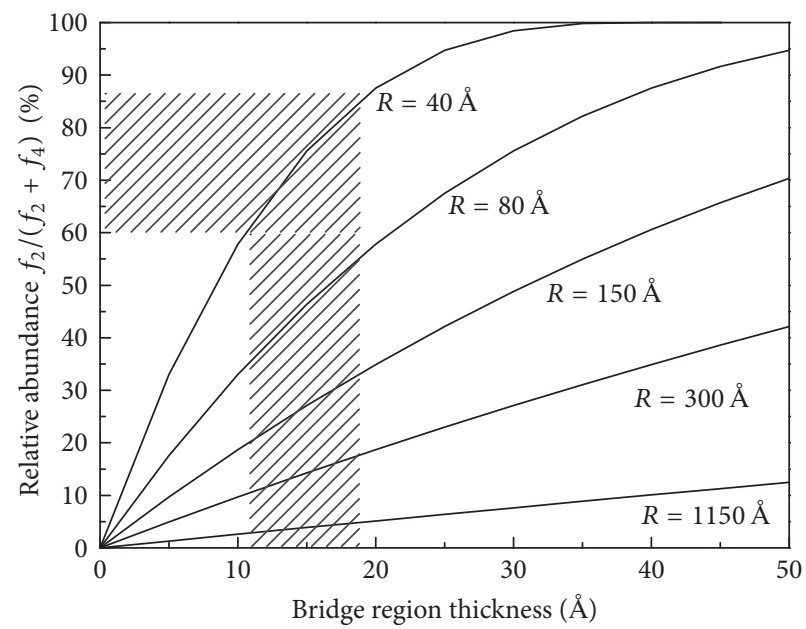

FIGURE 6: Relative abundances of the various surroundings of the PAC probe nuclei $f_{2} /\left(f_{2}+f_{4}\right)$ in the metamict state as a function of the bridge region thickness.

degree decreases from AUS, SUD, and USA natural sands. Besides, USA sands display better zircon crystallinity than the other two natural sands.

The results have confirmed that the metamict state of zircon natural sands is a dispersion of submicron domains in a crystalline matrix of zircon. The zircon sands contain depleted cavities of about $80 \AA^{3}$ while the metamict region has an extension of the order of $40 \AA$ consisting in defective regions. These regions present $\mathrm{Zr}$-rich and Si-rich zones separated by a thin interface or bridge region of approximately $15 \AA$ thick (2-3 zircon cell constants). 
The complete recovery of the zircon lattice after an annealing treatment was confirmed by XRD and PALS results. Instead, the very local technique (PAC) points out the existence of metamict residues in all the samples.

\section{Disclosure}

Present affiliation for Giorgia Cinelli is European Commission, Joint Research Centre, Directorate G, Nuclear Safety \& Security, Unit G.10, Knowledge for Nuclear Safety, Safeguards and Security, Via Enrico Fermi 2749, 21027 Ispra, Italy.

\section{Conflicts of Interest}

The authors declare that there are no conflicts of interest regarding the publication of this paper.

\section{Acknowledgments}

The authors are grateful to Endeka Ceramics (Modena, Italy) for providing the zircon sand samples used in the present study.

\section{References}

[1] S. Motoi, "Review of ceramic materials in zircon and zirconia system," Bulletin of the Ceramic Society of Japan, vol. 7, no. 3, pp. 162-168, 1972.

[2] M. Lynch, "Zircon opacifier markets and trends," Asian Ceramics, pp. 36-41, 2007.

[3] IAEA, "Radiation protection and NORM residue management in the zircon and zirconia industries," Tech. Rep. 51, International Atomic Energy Agency ed., Vienna, Austria, 2007.

[4] S. Ellsworth, A. Navrotsky, and R. C. Ewing, "Energetics of radiation damage in natural zircon $(\mathrm{ZrSiO} 4), "$ Physics and Chemistry of Minerals, vol. 21, no. 3, pp. 140-149, 1994.

[5] L. Bruzzi, M. Baroni, G. Mazzotti, R. Mele, and S. Righi, "Radioactivity in raw materials and end products in the Italian ceramics industry," Journal of Environmental Radioactivity, vol. 47, no. 2, pp. 171-181, 2000.

[6] J. M. Hanchar, Zircon, vol. 53 of Reviews in Mineralogy and Geochemistry, Mineralogical Society of America, USA, 1 edition, 2003.

[7] M. Zhang, E. K. H. Salje, I. Farnan et al., "Metamictization of zircon: Raman spectroscopic study," Journal of Physics Condensed Matter, vol. 12, no. 8, pp. 1915-1925, 2000.

[8] S. Ríos, T. Malcherek, E. K. Salje, and C. Domeneghetti, "Localized defects in radiation-damaged zircon," Acta Crystallographica Section B: Structural Science, vol. 56, no. 6, pp. 947-952, 2000.

[9] S. Ríos, E. K. H. Salje, M. Zhang, and R. C. Ewing, "Amorphization in zircon: evidence for direct impact damage," Journal of Physics Condensed Matter, vol. 12, no. 11, pp. 2401-2412, 2000.

[10] F. Poitrasson, J. M. Hanchar, and U. Schaltegger, "The current state and future of accessory mineral research," Chemical Geology, vol. 191, no. 1-3, pp. 3-24, 2002.

[11] A. Kaiser, M. Lobert, and R. Telle, "Thermal stability of zircon $\left(\mathrm{ZrSiO}_{4}\right)$," Journal of the European Ceramic Society, vol. 28, no. 11, pp. 2199-2211, 2008.

[12] W. J. Weber, R. C. Ewing, and L.-M. Wang, "The radiationinduced crystalline-to-amorphous transition in zircon," Journal of Materials Research, vol. 9, no. 3, pp. 688-698, 1994.
[13] M. Colombo, J. Chrosch, and E. K. H. Salje, "Annealing metamict zircon: a powder X-ray diffraction study of a highly defective phase," Journal of the American Ceramic Society, vol. 82, no. 10, pp. 2711-2716, 1999.

[14] Z. Mursic, T. Vogt, and F. Frey, "High-temperature neutron powder diffraction study of $\mathrm{ZrSiO}_{4}$ up to $1900 \mathrm{~K}$," Acta Crystallographica Section B, vol. 48, no. 5, pp. 584-590, 1992.

[15] F. Farges, "The structure of metamict zircon: a temperaturedependent EXAFS study," Physics and Chemistry of Minerals, vol. 20, no. 7, pp. 504-514, 1994.

[16] F. Farges and G. Calas, "Structural analysis of radiation damage in zircon and thorite: an X- ray absorption spectroscopic study," American Mineralogist, vol. 76, no. 1-2, pp. 60-73, 1991.

[17] K. Trachenko, M. T. Dove, and E. K. Salje, "Structural changes in zircon under $\alpha$-decay irradiation," Physical Review B, vol. 65, no. 18, Article ID 189902, 2002.

[18] H. Jaeger and S. P. McBride, "Perturbed angular correlation measurement of the electric field gradient at ${ }^{181} \mathrm{Ta}$ in $\mathrm{ZrSiO}_{4}$ and $\mathrm{HfSiO}_{4}$," Hyperfine Interactions, vol. 177, no. 1-3, pp. 51-56, 2007.

[19] H. Jaeger, M. P. Rambo, and R. E. Klueg, "Hyperfine interaction of metamict zircon studied by PAC spectroscopy," Hyperfine Interactions, vol. 136-137, no. 3-8, pp. 515-521, 2001.

[20] G. R. Gilmour, Gamma-Ray Spectrometry, John Wiley \& Sons, 2008.

[21] D. C. Stromswold, "Calibration facilites for borehole and surface environmental radiation measurements," Journal of Radioanalytical and Nuclear Chemistry Articles, vol. 194, no. 2, pp. 393401, 1995.

[22] B. H. Toby, "EXPGUI, a graphical user interface for GSAS," Journal of Applied Crystallography, vol. 34, no. 2, pp. 210-213, 2001.

[23] W. Brandt and A. Dupasquier, Positron Solid State Physics, North-Holland, Amsterdam, Netherlands, 1983.

[24] P. Kirkegaard and M. Eldrup, "Positronfit extended: a new version of a program for analysing position lifetime spectra," Computer Physics Communications, vol. 7, no. 7, pp. 401-409, 1974.

[25] H. Frauenfelder and R. M. Steffen, "Alpha, Beta- and Gammaray spectroscopy," in Angular Correlation, K. Siegbahn, Ed., p. 997, North-Holland, Amsterdam, Netherlands, 1965.

[26] R. Dogra, A. P. Byrne, and M. C. Ridgway, "The potential of the perturbed angular correlation technique in characterizing semiconductors," Journal of Electronic Materials, vol. 38, no. 5, pp. 623-634, 2009.

[27] N. M. Rendtorff, M. S. Conconi, E. F. Aglietti et al., "Phase quantification of mullite-zirconia and zircon commercial powders using PAC and XRD techniques," Hyperfine Interactions, vol. 198, no. 1, pp. 211-218, 2010.

[28] P. Montes-Navajas, L. C. Damonte, and H. García, "Positron annihilation lifetimes in cucurbiturils: evidence of internal inclusion of gold in CB[7]," ChemPhysChem, vol. 10, no. 5, pp. 812-816, 2009.

[29] P. Lacentre and M. C. Caracoche, "The effects of radiation damage and annealing on the microstructure of a natural zircon observed by TDPAC," Radiation Effects and Defects in Solids, vol. 129, no. 3-4, pp. 181-191, 1994.

[30] M. C. Caracoche, J. A. Martínez, A. F. Pasquevich, P. C. Rivas, E. Djurado, and F. Boulc'h, "PAC characterization of Gd and Y doped nanostructured zirconia solid solutions," Physica B: Condensed Matter, vol. 389, no. 1, pp. 98-102, 2007.

[31] D. Wiarda, M. Uhrmacher, A. Bartos, and K. P. Lieb, "Electric field gradients at ${ }^{111} \mathrm{Cd}$ in binary oxides," Journal of Physics: Condensed Matter, vol. 5, no. 25, article 002, pp. 4111-4124, 1993. 

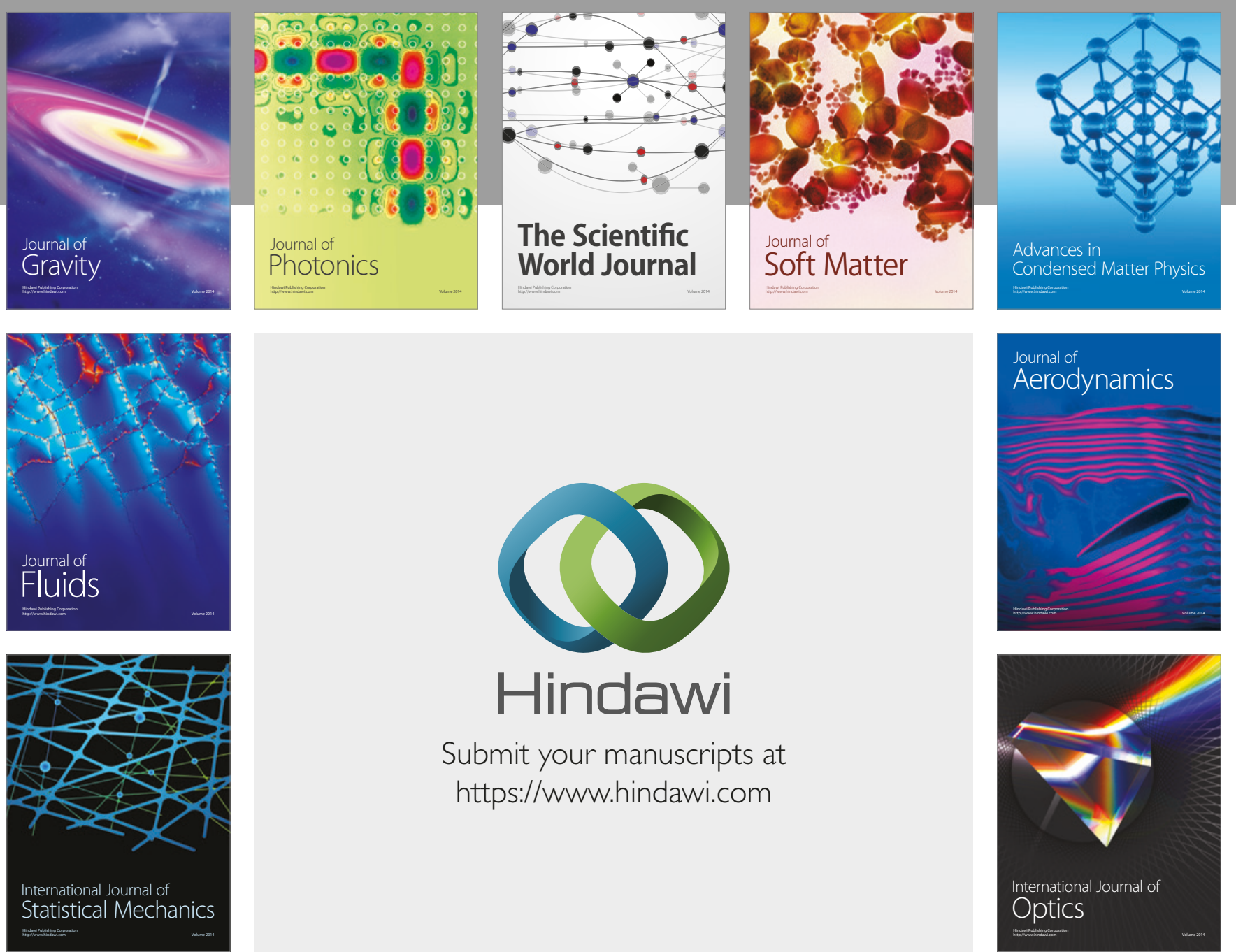

Submit your manuscripts at

https://www.hindawi.com
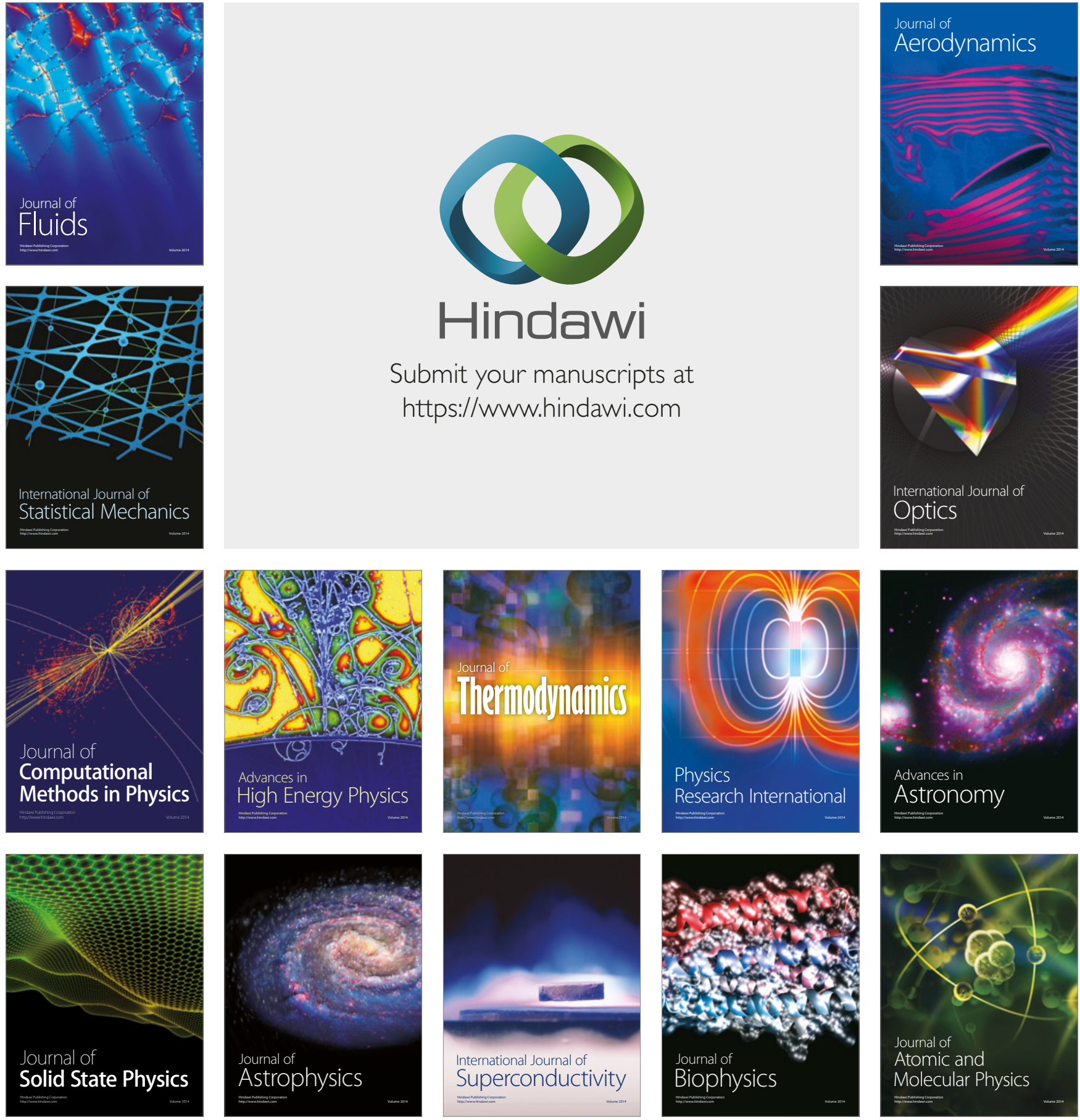